\title{
Friction weakening by mechanical vibrations: a velocity-controlled process
}

\author{
V. Vidal ${ }^{1 *}$, C. Oliver $^{2}$, H. Lastakowski ${ }^{1}$, G. Varas $^{2}$ \& J.-C Géminard ${ }^{1}$ \\ 1 Université de Lyon, Laboratoire de Physique, ENS de Lyon, CNRS, F-69342 Lyon, France and \\ 2 Instituto de Fisica, Pontificia Universidad Católica de Valparaiso, Av. Universidad 330, Valparaiso, Chile
}

(Dated: October 11, 2018)

\begin{abstract}
Frictional weakening by vibrations was first invoked in the 70's to explain unusual fault slips and earthquakes, low viscosity during the collapse of impact craters or the extraordinary mobility of sturzstroms, peculiar rock avalanches which travels large horizontal distances. This mechanism was further invoked to explain the remote triggering of earthquakes or abnormally large landslides or pyroclastic flows runout. Recent experimental and theoretical work pointed out the velocity of vibration as the key parameter which governs frictional weakening in sheared granular media. Here we show that the grains mobility is not mandatory, and that the vibration velocity governs both granular and solid frictional weakening. The velocity threshold controlling the transition from stickslip motion to continuous sliding is of the same order of magnitude, namely a hundred microns per second. It is linked to the roughness distribution of the asperities at the contact surface.
\end{abstract}

"It is easier to further the motion of a moving body than to move a body at rest." This sentence written by Themistius (about A.D. 320-390) is the first record of friction in history [1. Since then, the frictional motion of a single body over a fixed substrate or of a sheared granular assembly revealed a wide variety of behaviors. At low shear velocity, the system experiences a stick-slip motion, with the alternance of loading phases (system at rest) and quick slip phases which release the energy. When increasing the shear velocity, a transition to continuous sliding motion is reported 2, 5]. During catastrophic events such as earthquakes, landslides or pyroclastic flow, puzzling phenomena of frictional weakening were reported [6] 9]: friction decreases with the shear velocity. Melosh 10 first proposed in 1979 that vibrations due to particle collisions could temporarily reduce the normal stress, and thus decrease the shear stress threshold to trigger sliding motion [11. This mechanism, initially called vibrational fluidization and later on acoustic fluidization [10, 11, was further sought to be at the origin of dramatic events triggered by external waves, such as earthquake remote triggering [8, 12].

How does endogenous noise or external mechanical disturbances drastically affect the frictional properties? Many works have attempted to tackle this issue for the last decades. They have shown that vibrations reduce or even suppress friction 13 21. Interpretations were proposed based on a non-monotonic rheology curve, leading to instabilities and self-fluidization [22, 23], softening effect due to non-linearity at the grains contact [24, contact opening [25, 26] or sliding [27]. In single-block solid friction models, the vibration acceleration has often been stated at the parameter governing the transition between stick-slip motion and continuous sliding, with a threshold equal to the gravitational acceleration [19]. In a recent work, Lastakowski et al. 21] pointed out that the vibration velocity, and not the acceleration, is the parameter governing the frictional weakening in granu- lar assemblies. This result is independent of the slider velocity, contrary to what is expected from the classical rate-and-state heuristic model [3, 5, 28, 29. Surprisingly, the sheared granular exhibits a transition between stick-slip motion and continuous sliding for very low values of the vibration velocity, around $100 \mu \mathrm{m} / \mathrm{s}$, independently of most parameters which can be varied in the system (slider velocity, grain size and material, granular layer thickness, ...). A recent microscopic model based on sliding contacts under vibrations in a granular assembly successfully explains the dependence of the transition on the vibration velocity [27. However, important questions
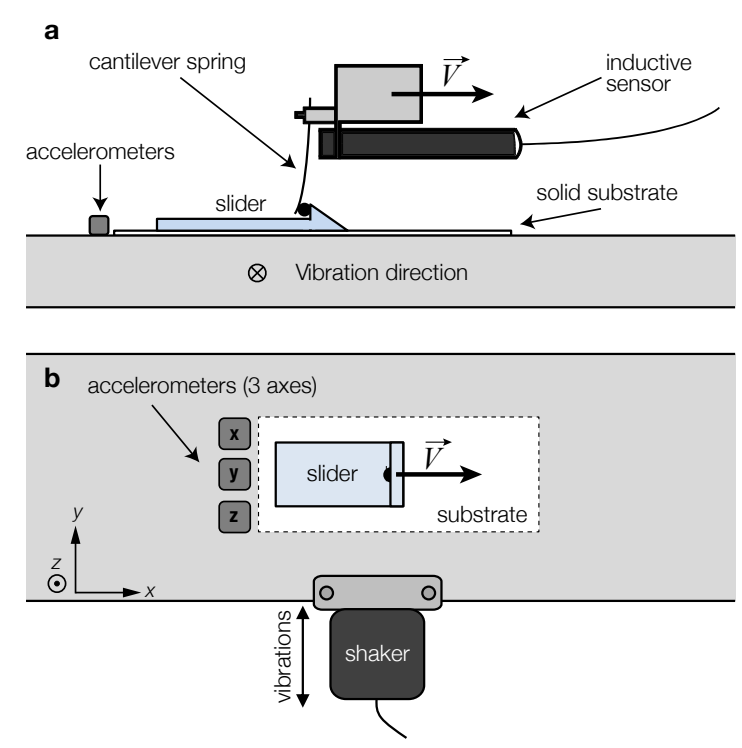

FIG. 1. Sketch of the experimental setup. (a) Side view. (b) Top view. Different substrates are used in the experiment. In the case of granular material, the accelerometers are located at the bottom of the granular layer, aligned with the shaker. 
arise: Is the grains mobility mandatory to experience this transition? Is vibration velocity the parameter governing the transition in solid friction too? Here we address these questions by studying experimentally solid (paper-paper) friction under harmonic vibrations.

Experimental setup - The setup is similar to the one used by Lastakowski et al. 21] (Fig. 1) to further compare the frictional weakening under vibrations in both solid and granular friction. It consists of a slider, made of plexiglas, of length $9 \mathrm{~cm}$ and width $6 \mathrm{~cm}$, pulled over a fixed substrate by means of a cantilever spring (metallic blade of stiffness $k$ ) (Fig. 1). A steel spherical ball is glued at the front of the slider to ensure a punctual contact so that no torque is applied to the slider. The blade is mounted on a translational stage (Schnaefler Technologies Sechnr) moving at constant velocity $V$. A DC motor (Crouzet, 5 N.m, $17 \mathrm{~W}$ ) coupled with differents reduction gears (Crouzet 1.04, 10, 100 RPM) allows to achieve velocities between 18-7700 $\mu \mathrm{m} / \mathrm{s}$. An inductive sensor (Baumer, IPRM 12I9505/S14) measures the blade deflection at a rate of $2 \mathrm{kHz}$. From the variations of the blade deflection in time, we derive the instantaneous force $F$ applied to the slider, and denote $F^{*}=F / m g$ the dimensionless force, where $m \simeq 22 \pm 5 \mathrm{~g}$ is the slider mass and $g=9.81 \mathrm{~m} \cdot \mathrm{s}^{-2}$ the gravitational acceleration.

Paper-paper friction is investigated by using two sets of samples: smooth printer paper (Inapa tecno copy/laser pro laser, $80 \mathrm{~g} / \mathrm{m}^{3}$, white) and rough drawing paper (Canson ${ }^{\circledR}$ Papier dessin blanc C grain, $180 \mathrm{~g} / \mathrm{m}^{2}$, rough surface). Reproducibility is systematically checked by performing different experiments for each given set of parameters. To avoid possible variations due to atmospheric conditions, the whole experiment is set inside a large box of controlled temperature $T$ and humidity $R_{H}$. For all experiments on paper-paper friction, $T=35 \pm 2^{\circ} \mathrm{C}$ and $R_{H}=20 \pm 2 \%$. Results for granular material are inferred from the analysis of the previous experimental data of Lastakowski et al. 21.

Vibrations are imposed to the whole experiment by a shaker (Brüel \& Kjær, type 4810 + amplifier 2706) clamped on the aluminum frame. It applied horizontal harmonic vibrations perpendicular to the slider's direction of motion, along the $y$-axis (Fig. 1). The vibrations amplitude $A$ and frequency $\omega$ are measured in situ close to the slider, at the surface of the solid substrate, by three accelerometers (Dytran Instrument, model \# 3035BG) getting the three components of acceleration. Before performing any experiment, we checked that the local acceleration is correctly oriented in the $y$-direction, and constant over a region large enough to include the slider motion.

Weakening and suppression of granular or solid friction - The pulling velocity $V$, slider mass $m$ and spring stiffness $k$ (Fig. 1) are chosen such that, in absence of vibrations, the slider always experiences a well-defined stick-slip motion, characterized by a saw-tooth shape of the instantaneous force applied to the slider (upper signal, insets Figs. 2a,b). At fixed vibration frequency
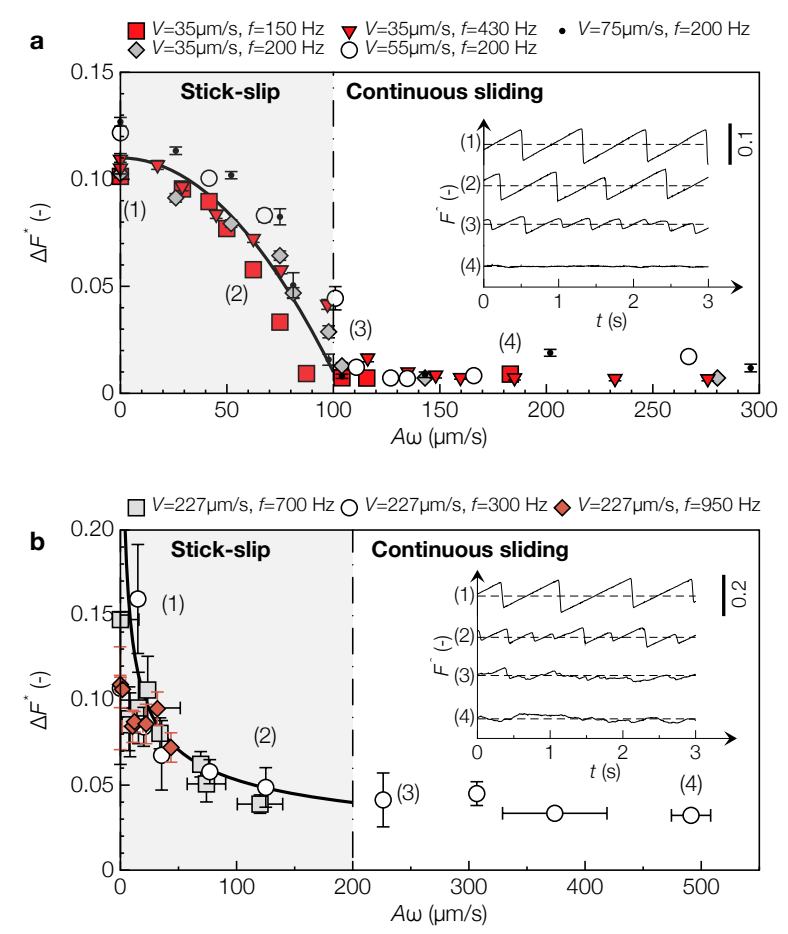

FIG. 2. Normalized force applied on the slider, $F^{*}$ for (a) granular and (b) solid (paper-paper, Inapa) friction [black lines are guides to the eye]. Insets: examples of normalized force as a function of time when increasing the vibration velocity, $(A \omega)$, for (a) granular $[k=870 \mathrm{~N} / \mathrm{m}, V=35 \mu \mathrm{m} / \mathrm{s}$, red squares in (a)] and (b) solid friction $[k=170 \mathrm{~N} / \mathrm{m}$, $V=227 \mu \mathrm{m} / \mathrm{s}$, white circles in (c)].

$f=\omega / 2 \pi$, when increasing the vibration amplitude, we observe a decrease of the normalized force signal amplitude, $\Delta F^{*}$ (insets Figs. 2a,b). Previous results on granular friction pointed out the vibration velocity, $(A \omega)$, as the governing parameter driving frictional weakening. Reanalysing data from Lastakowski et al. [21], we evidence this dependence on the stick-slip amplitude decay (Fig. 2a). Note the convex shape of the curve, independent of the pulling velocity as long as it is small enough for the system to be in stick-slip motion without vibrations. The well-marked transition, at a critical vibration velocity $(A \omega)_{c} \sim 100 \mu \mathrm{m} / \mathrm{s}$, was found independent of most experimental parameters (grain shape and material, pulling velocity, granular layer thickness, etc.) [21.

For solid friction, we also report a weakening and suppression of friction when increasing the vibration amplitude (Fig. $2 \mathrm{~b}$ ). The vibration velocity, $(A \omega)$, is also the governing parameter for the friction decay and suppression, independently of the pulling velocity $V$. Interestingly, some differences appear between solid and granular friction. For Inapa paper, the shape of the frictional weakening is concave, and does not display a clear transition between stick-slip and continuous sliding as in granular assemblies. The gray zone, which indicates the region 

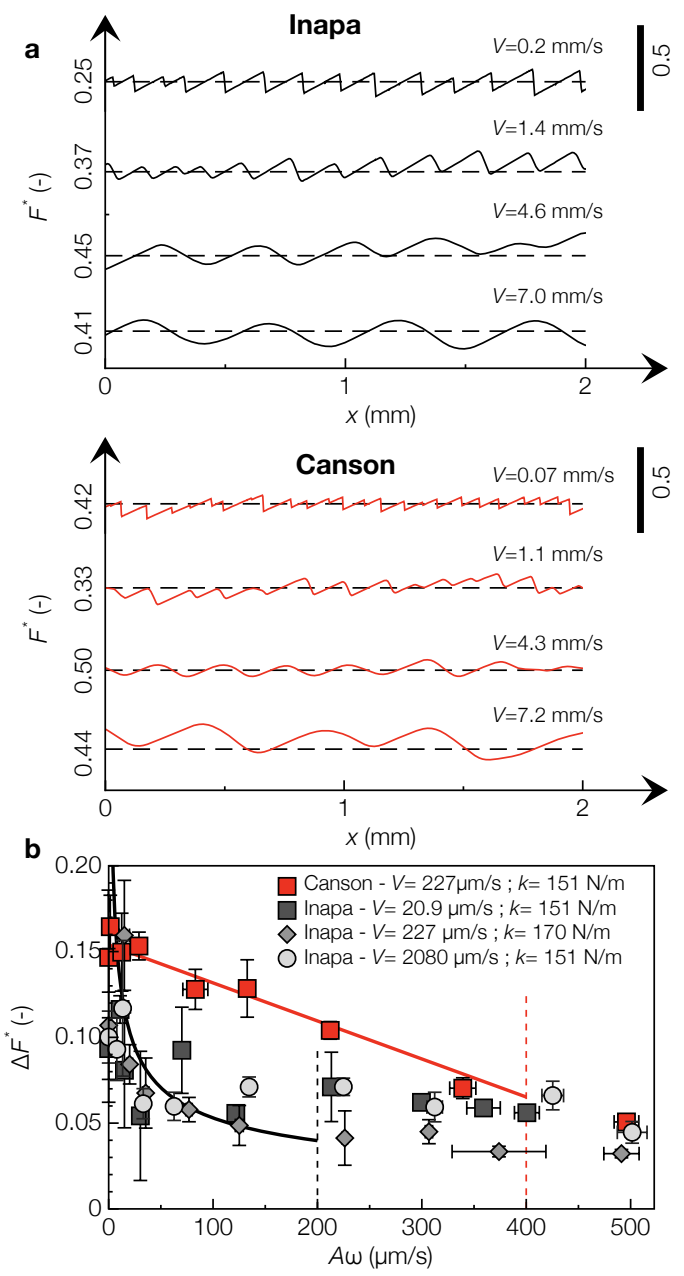

FIG. 3. Solid (paper-paper) friction dependence on pulling velocity or vibration velocity. (a) Normalized force $F^{*}$ as a function of time for different pulling velocity $V$, in absence of vibrations [up, Inapa paper; down, Canson ${ }^{\circledR}$ paper; $k=170 \mathrm{~N} / \mathrm{m}]$. The label on the vertical axes indicate the average value of the dimensionless force [dashed line]. (b) Normalized force variations amplitude $\Delta F^{*}$ for Inapa (gray and black) and Canson ${ }^{\circledR}$ (red) paper as a function of the vibration velocity, $(A \omega)$ [solid lines are guide to the eye, $f=300 \mathrm{~Hz}$ ].

where stick-slip motion occurs, is delimitated by checking the force signals (Fig. 2p, inset) and picking the vibration velocity above which the slider does not experience any more rest phases, i.e. linear increases in the force signal. The critical vibration velocity, although of the same order of magnitude than for granular friction, has increased by a factor 2 for Inapa paper, $(A \omega)_{c} \simeq 200 \mu \mathrm{m} / \mathrm{s}$.

Role of roughness in frictional weakening - First, the critical vibration velocity is compared to the slider velocity necessary to undergo the transition between stick-slip and continuous sliding in absence of vibrations. Fig. 3a (up) displays the normalized force exerted on the slider as a function of time for different pulling velocity $V$, in absence of vibrations $(A \omega)=0$. As predicted by a simple friction model [5], the slider first undergoes a transition between stick-slip motion and an inertial regime, characterized by a periodic motion without any more rest phases and a relatively large amplitude. However, no steady-sliding is observed in the experimental range of parameters, as it would require a much larger pulling velocity, which cannot be reached by our setup. Therefore, a simple force (or velocity) composition has to be discarded to explain the transition between stick-slip and continuous sliding when imposing vibration to the system. Second, the critical vibration velocity $(A \omega)_{c}$ to enter the steady sliding regime in granular assembly was previously explained as the critical energy to overcome a potential energy barrier, namely the typical size of an asperity at the grain surface 21. This explanation was in agreement with the independence of $(A \omega)_{c}$ on the grain shape or material (e.g. spherical glass beads or irregular ceramic beads), as the typical subscale of roughness was similar, at the nanometer scale.

To test this hypothesis, we performed additional experiments on Canson ${ }^{\circledR}$, a commercial paper which exhibits a rough surface traditionally used for charcoal drawing. We checked that for this paper, the pulling velocity alone is never enough to provoke the transition between stickslip motion and continuous sliding (Fig. 3a, down), as for Inapa paper. We then investigated the frictional weakening when imposing mechanical disturbances. One again, all data collapse when plotting the force variations amplitude, $\Delta F^{*}$, as a function of the vibration velocity, $A \omega$ (Fig. 3b). However, we observe a change in the critical vibration velocity to undergo the continuous sliding motion. For Canson ${ }^{\circledR}$ paper, indeed, $(A \omega)_{c} \simeq 400 \mu \mathrm{m} / \mathrm{s}$ (vertical red dashed line, Fig. 3b), representing an increase of about a factor 2 respect to Inapa paper. Note that similarly to granular assemblies, both the shape of the frictional weakening curve and the critical vibration velocity are robust for different spring constant $k$ and pulling speed $V$ for a given paper-paper friction (Fig. 3 b). Changing the paper surface properties, however, induces a change in the curve shape, which exhibits a linear decrease for Canson ${ }^{\circledR}$.

To further quantify the link with surface roughness properties, we performed Scanning Electron Microscopy (SEM, Supra 55, VP Zeiss) and Atomic Force Microscopy (AFM, NanoWizard ${ }^{\circledR}$ 4, JPK Instruments) measurements on Inapa and Canson ${ }^{\circledR}$ paper samples. Figures $4 \mathrm{a}, \mathrm{b}$ display SEM images of Inapa (Fig. 4a) and Canson ${ }^{\circledR}$ (Fig. 4p) paper, respectively. Although Canson ${ }^{\circledR}$ has a rougher surface, paper fibers are smaller than Inapa paper. This indicates that the paper fibers size is not the right scale of roughness, as they are most probably flattened during paper manufacturing. As already proposed for granular assemblies 21, the smaller scale of roughness may be the one controlling the friction. Smaller structures can be spotted on Figures $4 a, b$, which size is smaller than a few microns. To quantify the smaller scale of roughness, we then performed AFM measurements on both paper samples. Their topogra- 

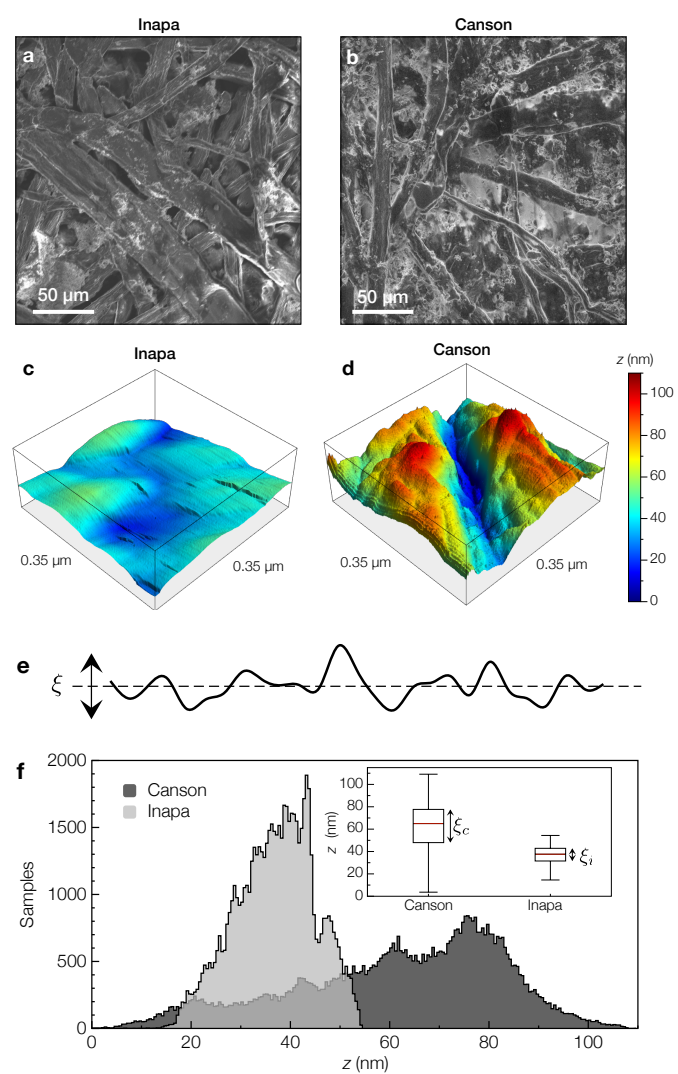

FIG. 4. Surface roughness analysis. (a,b) SEM images for Canson ${ }^{\circledR}$ (a) and Inapa (b) papers. (c,d) AFM topographic map of $0.35 \mu \mathrm{m} \times 0.35 \mu \mathrm{m}$ samples of Canson ${ }^{\circledR}$ (a) and Inapa (b) papers. The colorbar indicates the height in nanometers. (e) Schematic view of the dispersion for the smaller scale of roughness. The typical height of asperities is noted $\xi$. (f) Statistical distribution of the asperities size. Inset: Box plot representation. The box size represents the IQR (InterQuartile Range) and give the typical size of the asperities for the Canson ${ }^{\circledR}, \xi_{c}$, and Inapa, $\xi_{i}$, papers.

phy at small scale is clearly different, with rough linear structures for Canson ${ }^{\circledR}$ (Fig. 4 d) in contrast to smoother bumps for Inapa (Fig. 4k). The typical scale of the asperities, $\xi$ (Fig. 4e) is represented in the inset of Figure 4 for Canson ${ }^{\mathrm{B}}\left(\xi_{c}\right)$ and Inapa $\left(\xi_{i}\right)$ by the vertical size of the boxes in a box plot representation. We find $\xi_{c} \simeq 36.8 \mathrm{~nm}$ and $\xi_{i} \simeq 11.3 \mathrm{~nm}$. Following Lastakowski et al. 21] and DeGiuli \& Wyart [27, the energy of the external vibrations necessary to undergo the transition between stick-slip and continuous sliding should be of order $E_{e} \sim(A \omega)^{2}$, and comparable to the potential energy barrier to overcome, $E_{p} \sim \xi$. The critical velocity, $(A \omega)_{c}$, should therefore scale as the square root of the roughness, $\sqrt{\xi}$. Statistical analysis of the AFM measurements give the ratio between Canson ${ }^{\circledR}$ and Inapa paper samples, $\sqrt{\xi_{c} / \xi_{i}} \sim 1.8$, in agreement with the critical velocity ratio of about 2 for both solid substrates. In the same way, it also gives the typical asperity scale for the grains, $\xi_{g}=\xi_{i} / 4 \simeq 2.8 \mathrm{~nm}$, in agreement with Lastakowski et al. 21, who mentionned the typical size of an asperity at the grains surface to be of the order of a few nanometers.

Discussion - Numerical models for solid friction under vertical vibrations predicted a transition between stickslip and continuous sliding motion governed by the vibration acceleration, with a threshold at $A \omega^{2} \simeq g$, the gravitational acceleration [19. The above results demonstrate that, as previously shown in granular assemblies, the vibration velocity, and not acceleration, is the parameter controlling the transition. It is interesting to note that, for the typical frequency range used in the experiment, the threshold velocity corresponds to a very small value of the acceleration, typically a few percents of the gravitational acceleration.

For granular assemblies or, more generally, soft glassy materials, generic models have been proposed to predict the disappearance of the yield stress and trigger of a continuous motion or "flow" of the system 30. On the one hand, the classical trap model describes a particle in an energy landscape, where the external mechanical noise acts as an activation mechanism and has an equivalent in terms of effective temperature, even for athermal systems such as granular media 3134 . On the other hand, more recent models pointed out different statistical behaviours for thermal and athermal systems, and suggested that the mechanical noise would lead to a global inclination of the energy landscape, rather than an increase of the particle energy to escape the well in which it is trapped 35, 36. Recent experimental evidences on dry granular media have shown that small controlled mechanical fluctuations, whose amplitude is much smaller than the granular assembly yield stress, are enough to provoke a macroscopic flow by an accumulative process: tiny effects integrated over time can lead to the system fluidization, as a secular drift mechanism [37, 38. However, although the global effective rheology depends also, in this case, on the product between the vibration amplitude and the frequency - in other words, on the vibration velocity, the authors do not find any velocity threshold $(A \omega)_{c}$ as their system flows continuously for any tiny applied vibration. A possible explanation could be the existence of a smaller, microscopic scale in the energy landscape basins, as recently suggested by Charbonneau et al. [39], which were not captured by the previous experimental devices but successfully captured by our experiments.

Conversely, both numerical [40] and theoretical [27] works mention the existence of a vibration threshold below which no frictional weakening occur in granular assemblies, in agreement with laboratory fault-gouge experiments [8, 12, 41] and field measurements [42. Both high-pressure experiments and field data suggest an increase of this threshold velocity with the normal load - of the order of $1 \mathrm{~cm} / \mathrm{s}$ for $0.1 \mathrm{MPa}$ and $10 \mathrm{~cm} / \mathrm{s}$ for $10 \mathrm{MPa}$ fault load, for instance 42 .

In summary, one could suggest the existence of two thresholds: (1) a "high" threshold $(A \omega)_{c}$ above which 
the yield stress vanishes and the system experiences a continuous motion; (2) a "low"" threshold $(A \omega) *$ to trigger the flow, increasing with confining pressure - which was not captured by our experimental device. The difficulty in capturing both thresholds lies in measuring tiny effects, at the scale of asperities, together by increasing strongly the normal load (from 0.1 to $10 \mathrm{MPa}$ typically in fault gouges experiments or in the field [8, 12, 15, 42]). The experimental challenge is still open.
Acknowledgements - The authors thank Ludovic Bellon and Vincent Dolique for their help on SEM and AFM measurements. G.V. acknowledges financial support from PUCV DI Regular No. 039.438/2017. This work was supported by Programa de Cooperación Científica ECOS/CONICYT C14E07 and the Laboratoire International Associé "Matière: Structure et Dynamique" (LIAMSD, France-Chile).
[1] Sambursky, S. The Physical World of Late Antiquity (Princeton Univ. Press, 1962). 188 p.

[2] Baumberger, T., Heslot, F. \& Perrin, B. Cross-over from creep to inertial motion in friction dynamics. Nature $\mathbf{3 6 7}$, 544-546 (1994).

[3] Marone, C. Laboratory-derived friction laws and their application to seismic faulting. Ann. Rev. Earth Planet. Sci. 26, 643-696 (1998).

[4] Nasuno, S., Kudrolli, A., Bak, A. \& Gollub, J. P. Timeresolved studies of stick-slip friction in sheared granular layers. Phys. Rev. E 58, 2161-2171 (1998).

[5] Baumberger, T. \& Caroli, C. Solid friction from stickslip down to pinning and aging. Adv. Phys. 55, 279-348 (2006).

[6] Collins, G. S. \& Melosh, H. J. Acoustic fluidization and the extraordinary mobility of sturzstroms. J. Geophys. Res. 108, 2473 (2003).

[7] Lucas, A., Mangeney, A. \& Ampuero, J.-P. Frictional velocity-weakening in landslides on Earth and on other planetary bodies. Nature Comm. 5, 3417 (2014).

[8] Xia, K., Huang, S. \& Marone, C. Laboratory observation of acoustic fluidization in granular fault gouge and implications for dynamic weakening of earthquakes faults. Geochem. Geophys. Geosyst. 14, 1012-1022 (2013).

[9] Levy, C. et al. Friction weakening in granular flows deduced from seismic records at the Soufrire Hills Volcano, Montserrat. J. Geophys. Res. 120, 7536-7557 (2015).

[10] Melosh, H. J. Acoustic fluidization: A new geologic process? J. Geophys. Res. 84, 7513-7520 (1996).

[11] Melosh, H. J. Dynamic weakening of faults by acoustic fluidization. Nature 379, 601-606 (1996).

[12] Johnson, P. A. \& Jia, X. Nonlinear dynamics, granular media and dynamic earthquake triggering. Nature 437, 871-874 (2005).

[13] Rozman, M. G., Urbakh, M. \& Klafter, J. Controlling chaotic frictional forces. Phys. Rev. E 57, 7340-7343 (1998).

[14] Gao, J., Luedtke, W. D. \& Landman, U. Friction control in thin-film lubrication. J. Phys. Chem. B 102, 50335037 (1998).

[15] Johnson, P. A., Savage, H., Knuth, M., Gomberg, J. \& Marone, C. Effects of acoustic waves on stick-slip in granular media and implications for earthquakes. Nature $\mathbf{4 5 1}$, 57-61 (2008).

[16] Capozza, R., Vanossi, A., Vezzani, A. \& Zapperi, S. Suppression of friction by mechanical vibrations. Phys. Rev. Lett. 103, 085502 (2009).

[17] Capozza, R., Rubinstein, S. M., Barel, I., Urbakh, M. \& Fineberg, J. Stabilizing stick-slip friction. Phys. Rev. Lett. 107, 024301 (2011).
[18] Melhus, M. F. \& Aranson, I. S. Effect of vibration on solid-to-liquid transition in small granular systems under shear. Gran. Matt. 14, 151-156 (2012).

[19] Giacco, F., Lippiello, E. \& Pica Ciamarra, M. Solid-onsolid single-block dynamics under mechanical vibration. Phys. Rev. E 86, 016110 (2012).

[20] Giacco, F., Saggese, L., de Arcangelis, L., Lippiello, E. \& Pica Ciamarra, M. Dynamic weakening by acoustic fluidization during stick-slip motion. Phys. Rev. Lett. 115, 128001 (2015).

[21] Lastakowski, H., Géminard, J.-C. \& Vidal, V. Granular friction: Triggering large events with small vibrations. Sci. Rep. 5, 13455 (2015).

[22] Dijksman, J. A., Wortel, G. H., van Dellen, L. T. H., Dauchot, O. \& van Hecke, M. Jamming, yielding, and rheology of weakly vibrated granular media. Phys. Rev. Lett. 107, 108303 (2011).

[23] Wortel, G., Dauchot, O. \& van Hecke, M. Criticality in vibrated frictional flows at a finite strain rate. Phys. Rev. Lett. 117, 198002 (2016).

[24] Jia, X., Brunet, T. \& Laurent, J. Elastic weakening of a dense granular pack by acoustic fluidization: Slipping, compaction, and aging. Phys. Rev. E 84, 020301(R) (2011).

[25] DeGiuli, E., Düring, G. \& Wyart, M. Unified theory of inertial granular flows and non-Brownian suspensions. Phys. Rev. E 91, 062206 (2015).

[26] Ferdowsi, B. et al. Acoustically induced slip in sheared granular layers: Application to dynamic earthquake triggering. Geophys. Res. Lett. 42, 9750-9757 (2015).

[27] DeGiuli, E. \& Wyart, M. Friction law and hysteresis in granular materials. PNAS 114, 9284-9289 (2017).

[28] Gu, J.-C., Rice, J. R., Ruina, A. L. \& Tse, S. T. Slip motion and stability of a single degree of freedom elastic system with rate and state dependent friction. J. Mech. Phys. Solids 32, 167-196 (1984).

[29] Rice, J. R. \& Tse, S. T. Dynamic motion of a single degree of freedom system following a rate and state dependent friction law. J. Geophys. Res. 91, 521-530 (1986).

[30] Sollich, P., Lequeux, F., Hébraud, P. \& Cates, M. E. Rheology of Soft Glassy Materials. Phys. Rev. Lett. 78, 2020-2023 (1997).

[31] Bouchaud, J.-P. Weak ergodicity breaking and aging in disordered systems. J. Phys. I France 2, 1705-1713 (1992).

[32] Monthus, C. \& Bouchaud, J.-P. Models of traps and glass phenomenology. J. Phys. A: Math. Gen. 29, 3847-3869 (1996).

[33] Sollich, P. Rheology constitutive equation for a model of soft glassy materials. Phys. Rev. E 58, 738-759 (1998). 
[34] Bocquet, L., Colin, A. \& Ajdari, A. Kinetic theory of plastic flow in soft glassy materials. Phys. Rev. Lett. 103, 036001 (2009).

[35] Nicolas, A., Martens, K. \& Barrat, J.-L. Rheology of athermal amorphous solids: Revisiting simplified scenarios and the concept of mechanical noise temperature 107, 44003 (2014).

[36] Agoritsas, E., Bertin, E., Martens, K. \& Barrat, J.-L. On the relevance of disorder in athermal amorphous materials under shear. Eur. Phys. J. E 38, 71 (2015).

[37] Derec, C., Ajdari, A. \& Lequeux, F. Rheology and aging: A simple approach. Eur. Phys. J. E 2, 355-361 (2001).

[38] Pons, A., Amon, A., Darnige, T., Crassous, J. \& Clément, E. Mechanical fluctuations suppress the threshold of soft-glassy solids: The secular drift scenario. Phys. Rev. E 92, 020201(R) (2015).
[39] Charbonneau, P., Kurchan, J., Parisi, G., Urbani, P. \& Zamponi, F. Fractal free energy landscapes in structural glasses. Nature Comm. 5, 3725 (2014).

[40] Ferdowsi, B., Griffa, M., Guyer, R. A., Johnson, P. A. \& Carmeliet, J. Effect of boundary vibration on the frictionnal behavior of dense sheared granular layer. Acta Mech. 225, 2227-2237 (2014).

[41] Johnson, P. A. et al. Nonlinear dynamic triggering of slow slip on simulated earthquake faults with implications to Earth. J. Geophys. Res. 117, B04310 (2012).

[42] Gomberg, J., Reasenberg, P. A., Bodin, P. \& Harris, R. A. Earthquake triggering by seismic waves following the Landers and Hector Mine earthquakes. Nature 411, 462-466 (2001). 